\title{
Evidence for geomagnetic jerks in comprehensive models
}

\author{
Aude Chambodut* and Mioara Mandea ${ }^{\dagger}$ \\ Institut de Physique du Globe de Paris, 4 Place Jussieu, 75252 Paris Cedex 05, France
}

(Received August 18, 2004; Revised January 14, 2005; Accepted January 17, 2005)

\begin{abstract}
The rate of secular variation occasionally undergoes a sudden, sharp change, called a geomagnetic jerk. Such jerks have been detected in geomagnetic time series, centered-over the last four decades_around 1971, 1980, 1991, and 1999; others have been inferred from historical records. The geomagnetic jerks represent a reorganization of the secular variation, implying an internal origin, as established through spherical harmonic and wavelet analysis. However, some characteristics of jerks are not well understood. Here we estimate the occurrence dates for geomagnetic jerks, as they can be detected from a global geomagnetic model. This choice makes the present study novel, for two reasons. First, utilizing the comprehensive modelling approach allows for the use of a secular variation signal free of time-varying external fields and their corresponding induced counterpart, and observatory biases. Second, the model utilizes satellite data when available, in addition to observatory data. Indeed, POGO (1967 to 1971), MAGSAT (1979 to 1980), Ørsted (1999 to present time) and CHAMP (2000 to present time) satellite measurements help to separate the different magnetic sources. In this study the CM4 comprehensive model is used for a global search of geomagnetic jerks and their occurrence dates. Our first result indicates that found geomagnetic jerks might not have been worldwide in occurrence. Moreover, the obtained dates suggest that jerks detected in the CM4 model over the last four decades occurred not simultaneously but at slightly different times around 1971, 1980 and 1991.
\end{abstract}

Key words: Geomagnetic field, secular variation, geomagnetic impulses, comprehensive models.

\section{Introduction}

The magnetic field, observed on the surface of the Earth, is mainly generated in the external core. Known as the core field, this is the largest component of the magnetic field, which is believed to be caused by electrical currents flowing in the Earth's fluid outer core. In addition to sources in the Earth's core the magnetic field has a sizable contribution from the lithosphere and crust, both from induced magnetisation from the core field, and also from permanent remanant magnetisation (field frozen into rocks at the time of their formation). The external magnetic field is generated from magnetic sources outside the Earth, and is mainly produced by tidal motions in the ionosphere and interactions of the Earth's magnetosphere with the solar wind. Another source is the externally induced field by currents flowing within the Earth's crust and mantle.

The geomagnetic field varies on a large range of scales, both in space and time domains. The external fields vary on a much shorter time scale than the core field, temporal variation of which is known as the secular variation. Although the secular variation usually occurs as smooth time changes of the core field, episodes of abrupt changes have occurred in the past. These are known as geomagnetic jerks or geomagnetic impulses (Courtillot et al., 1978). A serious

\footnotetext{
*Now at Universität Potsdam, Germany.

${ }^{\dagger}$ Now at GeoForschungsZentrum Potsdam, Germany.
}

Copy right(c) The Society of Geomagnetism and Earth, Planetary and Space Sciences (SGEPSS); The Seismological Society of Japan; The Volcanological Society of Japan; The Geodetic Society of Japan; The Japanese Society for Planetary Sciences; TERRAPUB limitation regarding the investigation of these internal processes with time-scales of months to a few years is the effect of geomagnetic variations of external origin, since they contribute significantly on these time-scales. An immediate consequence is that some fundamental characteristics of a jerk are not completely identified and still not agreed upon by researchers; among those are occurrence dates, duration, and their global or local character.

The last topic has been addressed recently (Bloxham et al., 2002). They suggested that jerks can be explained by a simple model of core dynamics that includes torsional oscillations, and that these events are strongly dependent on the local magnetic field at the top of the core. To support this hypothesis Bloxham et al. (2002) referred to the secular variation of the magnetic field at Niemegk (Germany) and Macquarie Island (Australia) observatories for the period 1950 to 2001. Geomagnetic jerks can be clearly observed in the East component of the secular variation at Niemegk observatory. At Macquarie Island, which is approximately antipodal to Niemegk, the secular variation is much less apparent, with little or no evidence of geomagnetic jerks. The authors claimed that the fact that jerks are most readily observed at European observatories, are largely confined to one component of the field, and are abrupt, argues for a local origin, perhaps a magnetic field instability.

Clearly, the global or local character of geomagnetic jerks and their simultaneity or lack thereof are important features of these phenomena. In order to make a systematic study of jerks, without any a priori assumption on their existence, location or form, a wavelet analysis was applied to the monthly mean series provided by geomagnetic ob- 
servatories (Alexandrescu et al., 1995; Alexandrescu et al., 1996). One of the advantages of this analysis is the possibility to detect and date singularities in a signal, as impulses in monthly mean series. In Alexandrescu et al. (1996), it was shown that the so-called 1969 and 1978 events display an intriguing space-time behavior, consisting of an early occurrence in the Northern hemisphere, followed by a later one in the Southern hemisphere, generally speaking. In Nagao et al. (2002), jerks were detected objectively and automatically using the third order spline function for a geomagnetic time series trend model at a given observatory, and applying this model to monthly means of each local time obtained from hourly means at 124 observatories. The authors also showed that the occurrence time for an impulse is slightly different between the Northern and Southern hemispheres. A much easier method to determine the date when a jerk occurs is to approximate secular variation time series by straight lines and to consider the intersection point of such lines as the date of an event (Chau et al., 1981; Stewart and Whaler, 1992). This method was also applied by De Michelis et al. (1998) and their analysis also showed that on a global scale geomagnetic jerks are not simultaneous.

The above studies generally confirm that geomagnetic jerks are detected with a time lag of a few years in occurrence dates in the two hemispheres. However, such events have only been witnessed in ground-based observatories so far, because none have occurred during the effective life time of recent magnetic satellite missions (Ørsted or CHAMP). The lack of continuous satellite observations of the geomagnetic field is an important limitation in elucidating where and when a geomagnetic jerk has occurred. To overcome this problem we used a time varying core field model to describe geomagnetic jerks. Recently, two geomagnetic models covering long-time periods have been published. One of these models (Jackson et al., 2000) is based on a new compilation of historical observations, and covers four centuries. Recently, another available model has been produced by Sabaka et al. (2004) covering the time-span 1960-2002. Due to its advantages (see below) this model is chosen in the present investigation. The initial phase of this kind of comprehensive modeling was first reported in Sabaka and Baldwin (1993) and the second phase in Langel et al. (1996). A brief description of the last available model proposed by Sabaka et al. (2004) is given in the following.

The Comprehensive Models (CMs) are constructed from ground-based and satellite geomagnetic observations. The last published model, denoted CM4 is an extension of CM3 (Sabaka et al., 2002), and solves simultaneously for core and crustal field, for ionospheric and magnetospheric contributions (plus their induced counterparts), and for the toroidal magnetic field produced by electrical currents at satellite altitude. The internal (core and lithospheric) fields are represented by a degree and order 65 internal spherical harmonic expansion, with the secular variation represented by cubic B-splines through degree and order 13 and a knot spacing of $2.5 \mathrm{yr}$, and extended through mid-2002. The CM4 model has been derived from the observatory hourly means closest to 01:00 h local time on the quietest day per month (1960-2000) and hourly means every $2 \mathrm{hr}$ on these quiet days during the POGO and MAGSAT missions. The quiet time POGO (1967-1971), MAGSAT (1979-1980), Ørsted (1999-2002), and CHAMP (2001-2002) satellite observations are also used. The use of ground-based and satellite data together facilitates separation of the various field sources and their spatial and temporal sampling extents. This is especially helpful when considering, as in the present paper, a core source for geomagnetic jerks. The comprehensive modeling approach proposed by Sabaka et al. (2004) presents two major advantages for our analysis, as it covers a recent period of time, when jerks are already well-detected in observatory data and fits the observatory data in their finer temporal details.

In the following we illustrate how well the CM4 model estimates the geomagnetic data by comparing the monthly means provided by the magnetic observatories and those computed from this model for the observatory locations. Thereafter, we search for geomagnetic jerks in time series of monthly means provided by observatories and in synthetic data computed all over the globe. We focus on the three well-documented last jerks, detected by Le Huy et al. (1998) in 1969, 1979 and 1992. Finally, maps indicating jerk occurrence dates over the 1960-2002 time-span are presented and discussed.

\section{Data}

Two datasets are used in this study. The first one is composed of the monthly mean series provided by magnetic observatories distributed worldwide ${ }^{1}$. The length of these series is different from one observatory to the other; but for the period we are interested in, we updated the existing series with the most recent published data, getting monthly means up to the end of 2003. The same wavelet analysis as in Alexandrescu et al. (1995) was carried out on this dataset in order to determine the occurrence dates for different events.

The second dataset consists of synthetic data. To estimate the magnetic field components at a given location for a given epoch and for given magnetic conditions (here quiet conditions), we used the CM4 associated code. This code returns the local $X, Y, Z$ (North, East, Vertical down) components of the $\vec{B}$ field of the various contributions (core, crust, primary magnetospheric, secondary (induced) magnetospheric, primary ionospheric, secondary (induced) ionospheric, toroidal magnetic field due to in-situ radial currents at satellite altitude). In our study only the core field is considered by using the spherical harmonic expansion up to degree/order 13 .

To analyze geomagnetic jerks it is essential to check how well the CM4 model fits the data provided by observatories. Available observatory monthly means for $X, Y$, and $Z$ field components are compared with the synthetic means estimated for the observatory locations. For this comparison all observatories providing minimum five-year series between 1960 and 2002 are kept. This amounts to 146 observatories shown in Fig. 1.

In the following examples of comparisons between real and synthetic monthly means for six observatories denoted

${ }^{1}$ http://obsmag.ipgp.jussieu.fr 


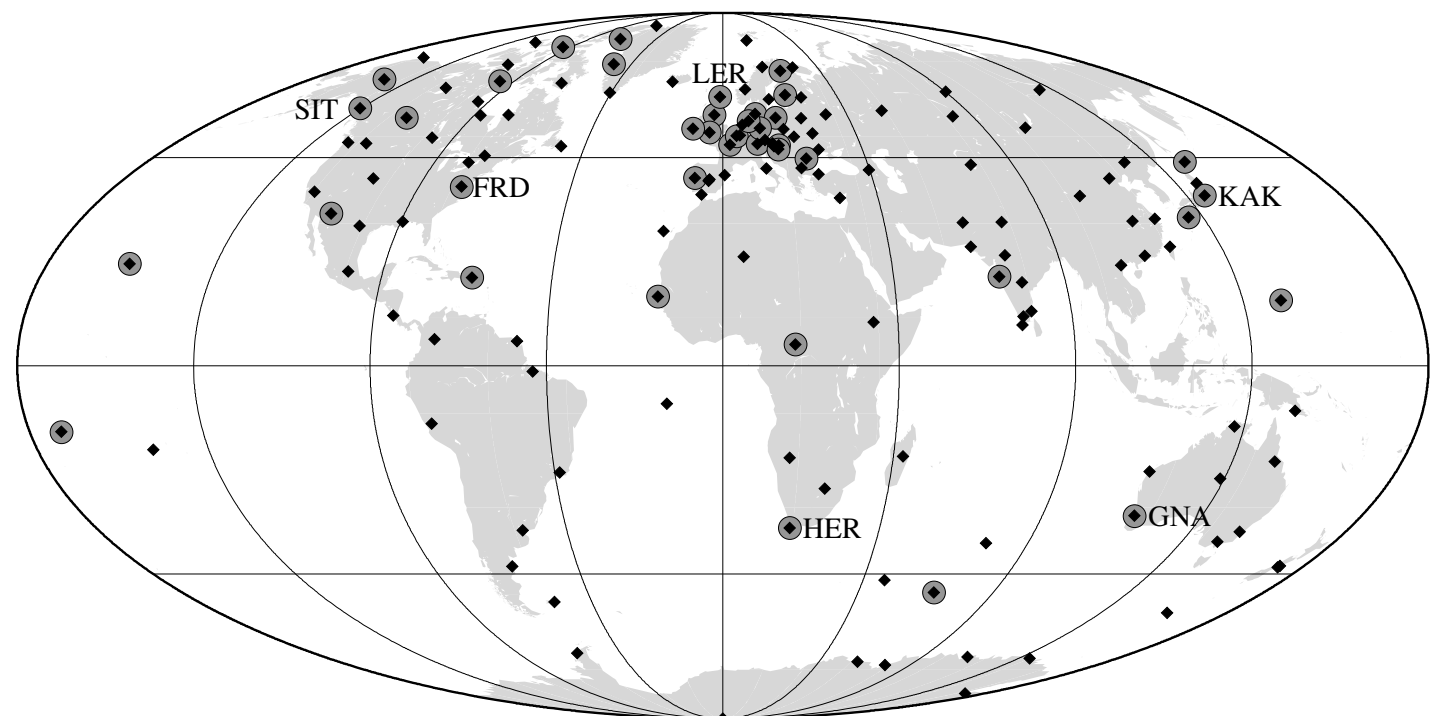

Fig. 1. Distribution of observatories used in the present study. Black diamonds: 146 observatories with minimum five-year monthly means between 1960 and 2002; gray circles: 39 observatories with continuous monthly mean series between 1960 and 2002. The IAGA codes are given for the six observatories chosen as examples.

Table 1. Crustal biases comparisons.

\begin{tabular}{|c|c|c|c|c|c|c|c|}
\hline & Code $^{\mathrm{a}}$ & $\lambda^{\mathrm{b}}$ & $\varphi^{\mathrm{c}}$ & model & $X_{c c}{ }^{\mathrm{d}}$ & $Y_{c c}{ }^{\mathrm{e}}$ & $Z_{c c}{ }^{\mathrm{f}}$ \\
\hline \multirow{3}{*}{ Fredericksburg } & \multirow{3}{*}{ FRD } & \multirow{3}{*}{38.210} & \multirow{3}{*}{282.630} & $M$ & 48 & -75 & 131 \\
\hline & & & & $\varnothing$ & 38 & -59 & 142 \\
\hline & & & & CM4 & 40 & -55 & 114 \\
\hline \multirow{3}{*}{ Gnangara } & \multirow{3}{*}{ GNA } & \multirow{3}{*}{-31.783} & \multirow{3}{*}{115.950} & $\mathrm{M}$ & -3 & -135 & 108 \\
\hline & & & & $\varnothing$ & -37 & -107 & 102 \\
\hline & & & & CM4 & -22 & -110 & 98 \\
\hline \multirow{3}{*}{ Hermanus } & \multirow{3}{*}{ HER } & \multirow{3}{*}{-34.425} & \multirow{3}{*}{19.225} & $\mathrm{M}$ & 19 & 13 & 10 \\
\hline & & & & $\varnothing$ & 3 & 15 & 11 \\
\hline & & & & CM4 & 3 & 12 & 30 \\
\hline \multirow{3}{*}{ Kakioka } & \multirow{3}{*}{ KAK } & \multirow{3}{*}{36.230} & \multirow{3}{*}{140.190} & $\mathrm{M}$ & -26 & 7 & -83 \\
\hline & & & & $\varnothing$ & -26 & 2 & -85 \\
\hline & & & & CM4 & -20 & 7 & -90 \\
\hline \multirow{3}{*}{ Lerwick } & \multirow{3}{*}{ LER } & \multirow{3}{*}{60.133} & \multirow{3}{*}{358.817} & $\mathrm{M}$ & -123 & 172 & 34 \\
\hline & & & & $\varnothing$ & -133 & 164 & 55 \\
\hline & & & & CM4 & -132 & 173 & 47 \\
\hline \multirow{3}{*}{ Sitka } & \multirow{3}{*}{ SIT } & \multirow{3}{*}{57.058} & \multirow{3}{*}{224.675} & M & -12 & -19 & -49 \\
\hline & & & & $\varnothing$ & -11 & -14 & -69 \\
\hline & & & & CM4 & -2 & -20 & -61 \\
\hline
\end{tabular}

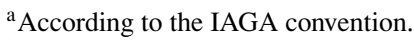

${ }^{\mathrm{b}}$ Latitude of the observatory, in degrees.

${ }^{\mathrm{c}}$ Longitude of the observatory, in degrees, positive eastward.

${ }^{\mathrm{d}, \mathrm{e}, \mathrm{f}}$ Crustal biases, using MAGSAT model (M), Ørsted model (Ø), and CM4 model (CM4), in nT.

by their IAGA codes (FRD, GNA, HER, KAK, LER, SIT) and located in different regions (see Fig. 1 and Table 1) are given. Figure 2 shows that some nearly-constant differences appear in synthetic values with regard to the real ones (see for example, the $X$ component for LER, $Y$ for GNA or LER, and $Z$ for GNA, KAK and SIT). They are due to crustal biases, as only the core field at the observatory locations is computed. The differences observed in Fig. 2 agree well with the published crustal biases (Mandea and Langlais, 2002), as shown in Table 1.

One simple way to avoid the crustal field signature is to directly estimate and analyse secular variation, computed here as the centered difference between two consecutive values:

$$
\dot{Y}\left(t_{i+\frac{1}{2}}\right)=\frac{Y\left(t_{i+1}\right)-Y\left(t_{i}\right)}{t_{i+1}-t_{i}} .
$$

For secular variation comparison only observatories with continuous series between 1960 and 2002 are kept: only 39 out of the 146 observatories providing data in this time span meet this requirement (Fig. 1). For these 39 observatories the secular variation has been computed, and there- 
FRD
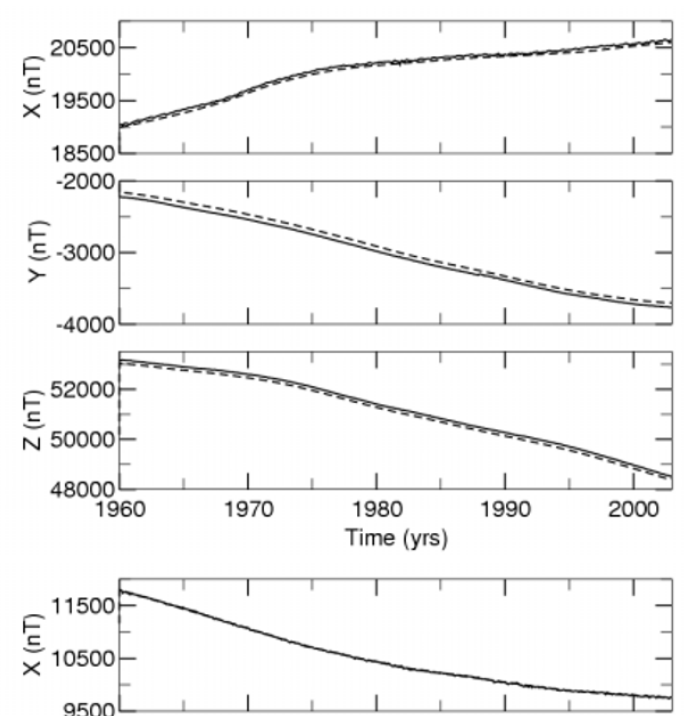

HER
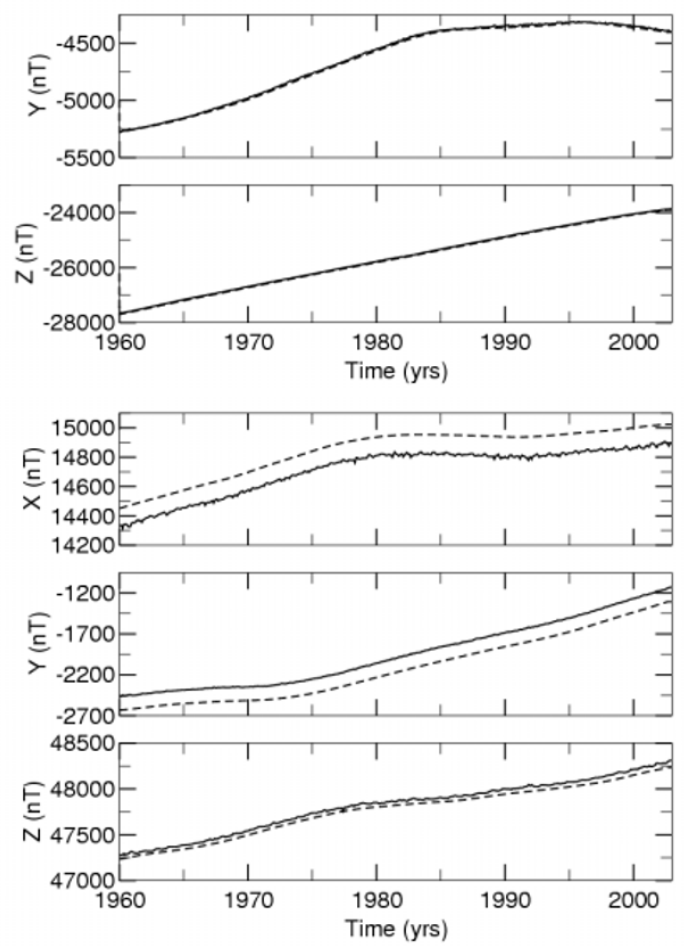

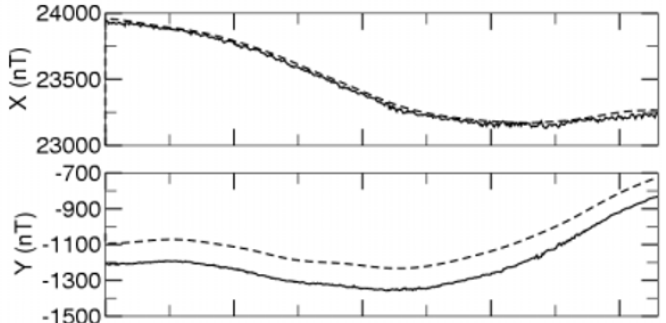

GNA
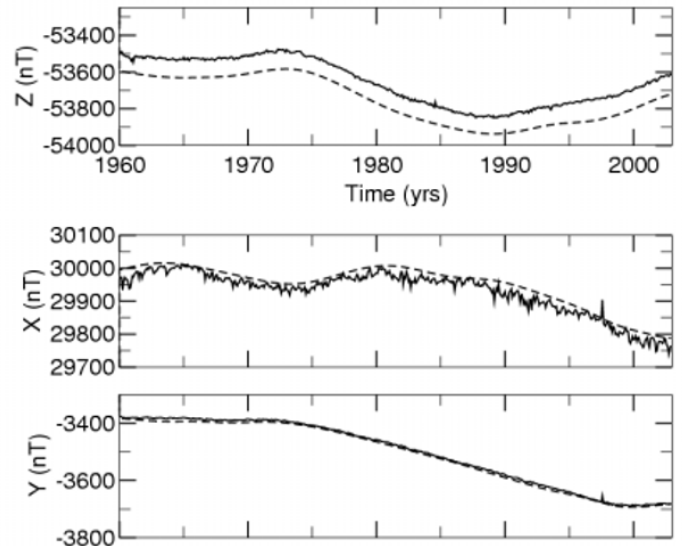

KAK
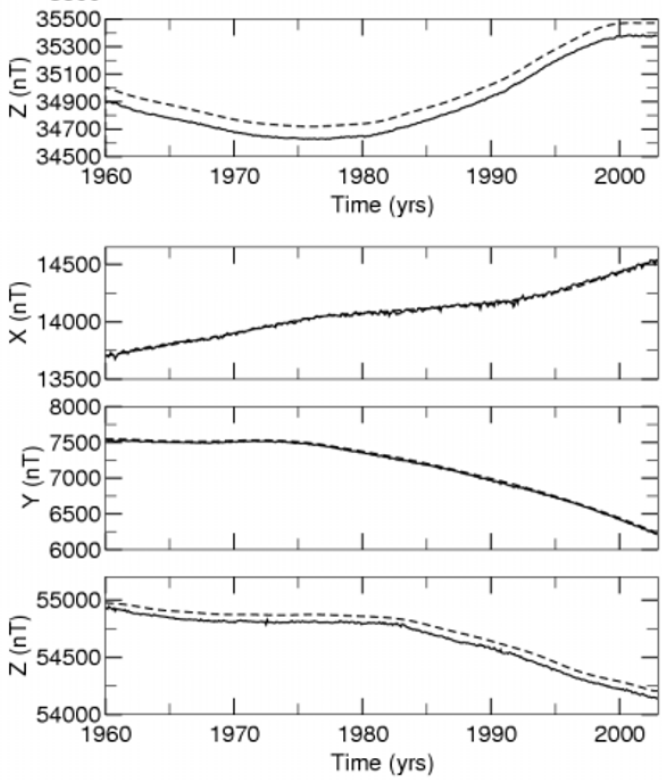

Fig. 2. Monthly means for the $X, Y$ and $Z$ field components (solid curve: real data, dashed curve: synthetic data given by the CM4 model).

after the $\dot{Y}\left(t_{i+\frac{1}{2}}\right)$ series have been smoothed with a simple 12-month running average to get rid of most of the external disturbance fields. The results obtained for the same six observatories are presented in Fig. 3. The secular variation comparison also shows the good agreement between real data and synthetic data computed from CM4 model. The resemblance, although imperfect - and there is little reason to expect it to be perfect as the core field computed from CM4 model is compared with real data containing all sources-is striking. The jerks are particularly clear in the $Y$ component of the secular variation, which is supposed to be the least affected by the external disturbance fields.

\section{Method of Analysis}

In the following, in order to estimate the secular variation trend for each point on the Earth's surface, we assume that in a given time interval $\left[t_{\text {begin }}, t_{\text {end }}\right]$, a geomagnetic jerk occurs at time $t_{0}$. We need some $a$ priori information about the mean epoch for each event and the choice of the $\left[t_{\text {begin }}, t_{\text {end }}\right]$ interval. To find the mean epoch for each event we considered the results obtained in detection and characterisation of geomagnetic jerks in observatory monthly series using the wavelet analysis method. Indeed, the mean epochs for the 1969 and 1978 jerks have been determined from Alexandrescu et al. (1996), where a clearly bimodal distribution of the dates of occurrence for these events is shown. For the so-called 1969 event a first group of dates is centered on $1969.5 \pm 0.5$ and another one $1972.1 \pm 0.5$; their merging date being around 1971. The dates for 1978 event split into a first group centered on $1977.9 \pm 0.6$ and a second one centered on $1981 \pm 0.5$, with a merging date around 1980 . For the 1991 jerk, we applied the same wavelet analysis 
FRD
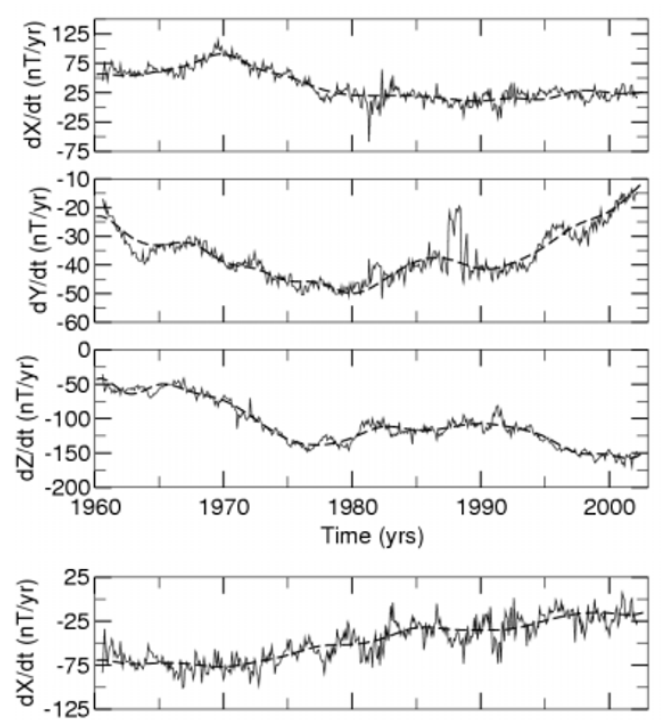

HER
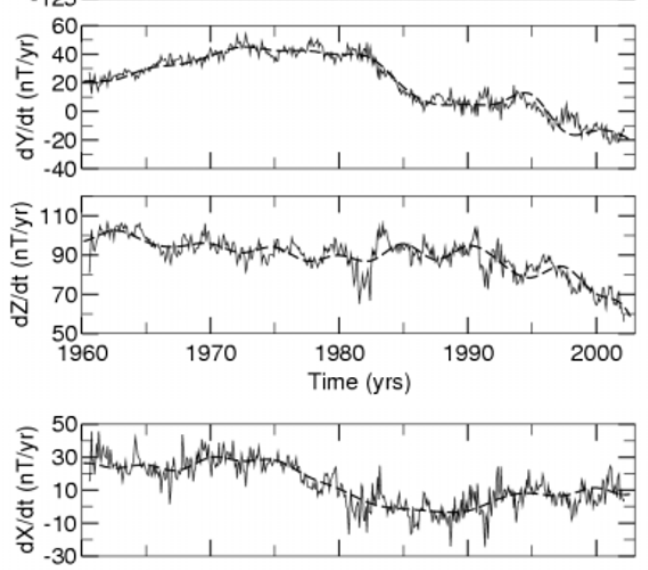

LER
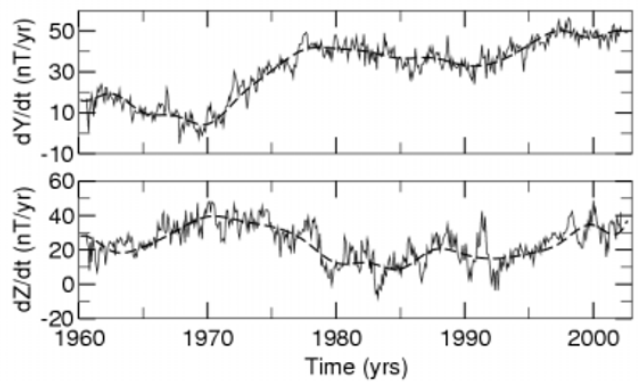
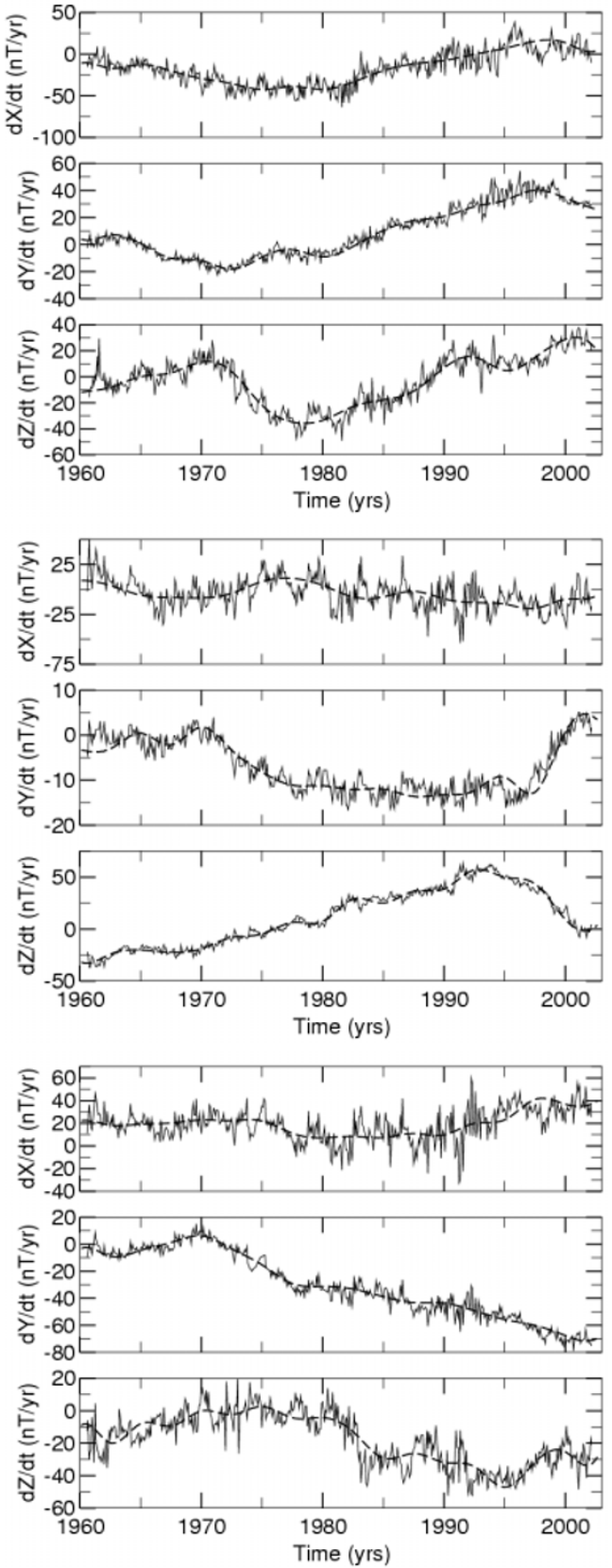

KAK

GNA

Fig. 3. Monthly means for $X, Y$ and $Z$ secular variation components (solid curve: real data, dashed curve: synthetic data given by CM4 model).

to monthly mean series beyond 2000 and found the mean epoch at $1990.6 \pm 1.6 \mathrm{yr}$. Thus, we consider 1991 the center of the third time interval.

The time interval has been chosen as twice the maximum time lag in the occurrence dates (Alexandrescu et al., 1996), $\left[t_{\text {begin }}, t_{\text {end }}\right]=6 \mathrm{yr}$. This a priori information is used in the following algorithm.

For the full interval covered by CM4 model we consider the time-span 1965-2000. The first five years are not kept for the analysis as the synthetic series seems to be more noisy. The year 2000 is the end of the studied interval, because of possible jerk at the end of the 20th century (Mandea et al., 2000). So, we focus on the three so-called 1969 , 1978 , and 1991 events. Each of these jerks is searched separately, using the best fit by piecewise linear functions over the four intervals. The first jerk occurring at $t_{0_{1}}$ is searched within the first interval $\left[t_{\text {begin }}, t_{\text {end }}\right]=[1968,1974]$. The $\dot{Y}$ secular variation component is approximated by two straight-line segments (from 1965 to 1977, the beginning years for the real or synthetic monthly series, and for the second interval containing a jerk, respectively) which best fit the data in the least-squares sense before and after the date $t_{0_{1}}$ :

$$
\begin{array}{ll}
\dot{Y}(t)=a_{1} t+b_{1} & \text { for: } t \leq t_{0_{1}}, \\
\dot{Y}(t)=a_{2} t+b_{2} & \text { for: } t \geq t_{0_{1}} .
\end{array}
$$

To determine the occurrence time $t_{0_{1}}$ of a jerk, the algorithm searches for a clear change of the slope in the straight-line segments. Thereafter the algorithm finds the best estimate of the secular variation by two straight-line segments (in the RMS sense). For example, for the first line segment, the 


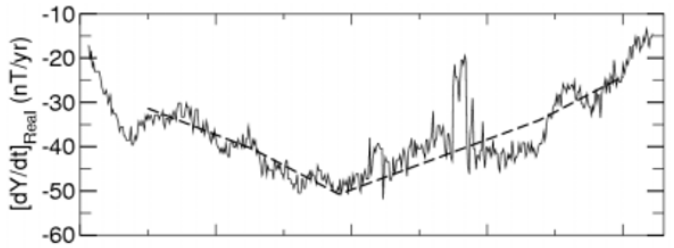

FRD
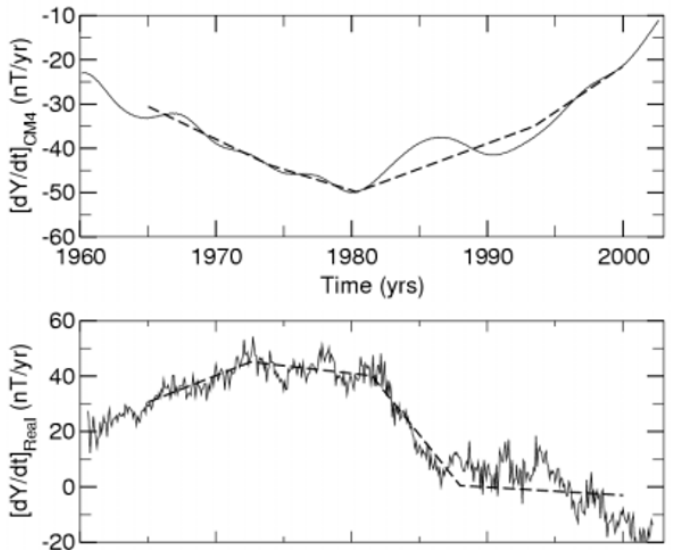

HER
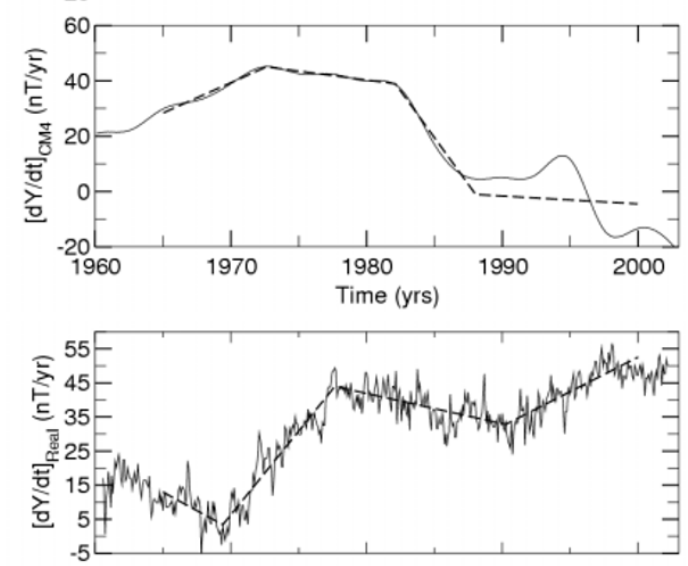

LER

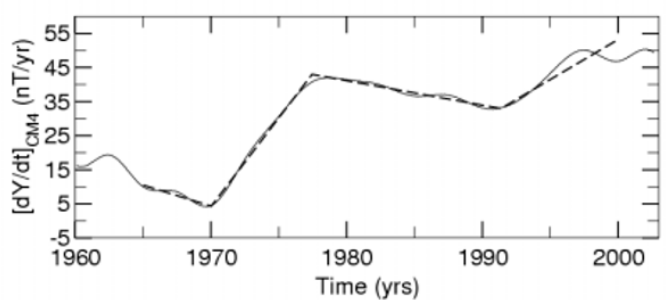

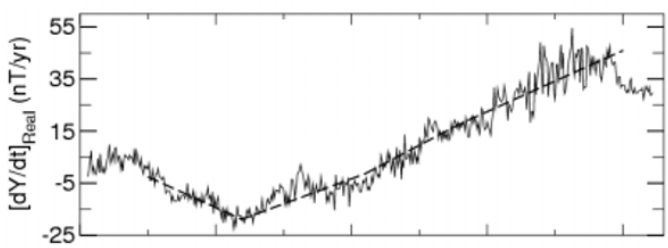

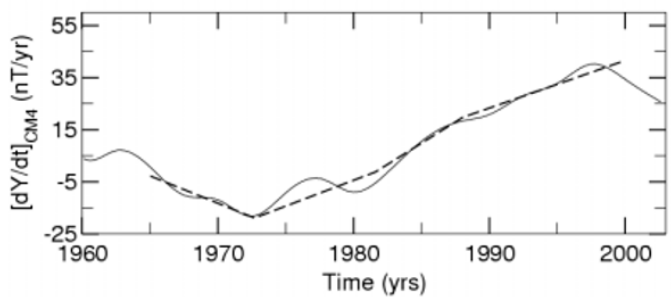

GNA
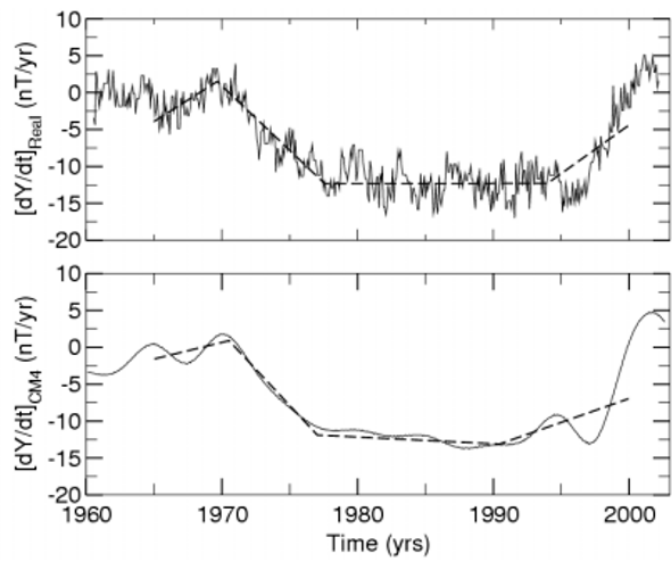

KAK
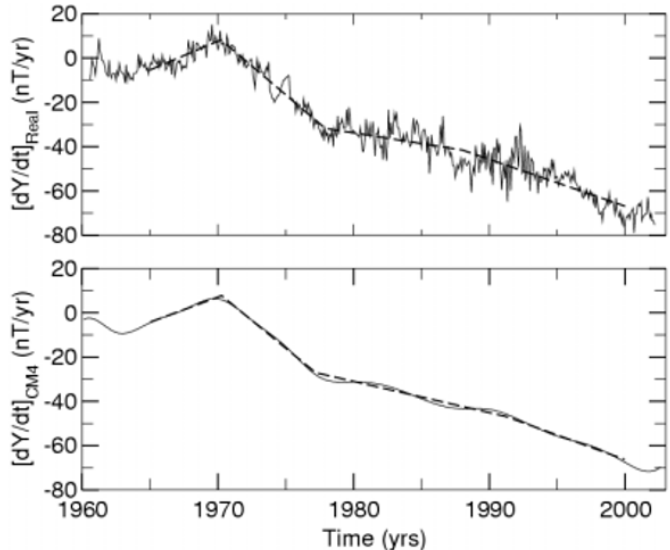

Fig. 4. Monthly means for $Y$ secular variation component. For each observatory the upper panel represents the linear approximation (dashed curve) of real data (solid curve); the lower panel represents the linear approximation (dashed curve) of synthetic data given by the CM4 model (solid curve).

quantity to be minimized is:

$$
\frac{\sum_{t}\left|\dot{Y}(t)-\left(a_{1} t+b_{1}\right)\right|}{N b} \longrightarrow \text { minimum }
$$

where $1965 \leq t \leq t_{0_{1}}$ and $N b$ the number of values on this interval.

The same method is applied for the other two investigated intervals. For the second one the chosen interval is $\left[t_{\text {begin }}, t_{\text {end }}\right]=[1977,1983]$ and the straight-line segments have to give the best fit between the first obtained occurrence date $t_{0_{1}}$ and the beginning of the third interval, i.e. 1988. The last time-span over which a jerk has been searched is $\left[t_{\text {begin }}, t_{\text {end }}\right]=[1988,1994]$. Finally, three occurrence dates $t_{0_{1}}, t_{0_{2}}$ and $t_{0_{3}}$ could be obtained for characterizing the three considered jerks.

\section{Results}

\subsection{Analysis at observatory locations}

The linear approximation method is applied to the secular variation of the $Y$ component for the 50 observatories, providing continuous monthly means between 1965-2000. We consider it useful to show again a few comparisons between the results obtained when using real and synthetic data. Figure 4 shows, for the same six observatories, the secular variation from the real data and its approximation by straight-line segments (upper panel for each observatory), and the secular variation estimated from the CM4 model and its corresponding approximation (lower panel).

FRD and GNA are situated at nearly antipodal locations. For both observatories only one event is very well-defined, the two others being more difficult to be defined. For FRD, 
Table 2. Date of occurrence of geomagnetic jerks (year/month).

\begin{tabular}{|c|c|c|c|c|}
\hline Code $^{a}$ & Event $^{\mathrm{b}}$ & Wavelet $^{\mathrm{c}}$ & Linear (real data) ${ }^{\mathrm{d}}$ & Linear (synthetic data) \\
\hline \multirow{3}{*}{ FRD } & 1 & $1969 / 06$ & N.D.* & N.D. \\
\hline & 2 & $1978 / 02$ & $1979 / 01$ & $1980 / 04$ \\
\hline & 3 & 1993/08 & N.D. & N.D. \\
\hline \multirow{3}{*}{ GNA } & 1 & $1972 / 08$ & $1971 / 11$ & $1972 / 09$ \\
\hline & 2 & $1980 / 12$ & N.D. & N.D. \\
\hline & 3 & N.D. & N.D. & N.D. \\
\hline \multirow{3}{*}{ HER } & 1 & 1972/06 & $1972 / 07$ & 1972/07 \\
\hline & 2 & 1982/01 & 1981/06 & 1982/01 \\
\hline & 3 & $1987 / 05$ & $1988 / 01$ & 1988/01 \\
\hline \multirow{3}{*}{ KAK } & 1 & $1969 / 06$ & $1969 / 08$ & 1970/07 \\
\hline & 2 & 1977/11 & 1977/06 & 1977/01 \\
\hline & 3 & N.D. & N.D. & N.D. \\
\hline \multirow{3}{*}{ LER } & 1 & $1969 / 02$ & $1969 / 05$ & 1970/01 \\
\hline & 2 & 1977/12 & 1977/08 & 1977/06 \\
\hline & 3 & $1990 / 04$ & $1990 / 03$ & 1991/05 \\
\hline \multirow{3}{*}{ SIT } & 1 & $1969 / 09$ & $1970 / 03$ & $1970 / 04$ \\
\hline & 2 & 1977/09 & $1977 / 12$ & 1977/03 \\
\hline & 3 & N.D. & N.D. & N.D. \\
\hline
\end{tabular}

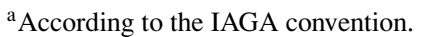

${ }^{\mathrm{b}}$ Considered geomagnetic jerks around: 1971 (1), 1980 (2) and 1991 (3).

${ }^{\mathrm{c}}$ Date of occurrence given by the wavelet analysis on real $Y$ data.

${ }^{\mathrm{d}}$ Date of occurrence given by the linear approximation on real $\dot{Y}$ data.

${ }^{\text {e }}$ Date of occurrence given by the linear approximation on synthetic $\dot{Y}$ data.

*N.D.-Not Detected.

the first and third events are not detected (the series provided by this observatory has an unusual behaviour between 1987-1988). At GNA observatory, the signature of the external field makes it difficult to identify the two last geomagnetic jerks. HER observatory is situated in an area with a considerable secular variation rate. Since the establishment of this observatory in 1941, the total field intensity has decreased by $20 \%$, which is larger than the decrease at any other magnetic observatory. Even in this very dynamic region approximation by straight-line segments is similar for real and synthetic data.

In the Northern hemisphere, KAK observatory is located in an area with less secular variation. The first two impulses are well-defined, the last one is suffering from the superimposed external contributions. LER observatory is a typical example for a region where all three events have been well determined. Indeed, in the European area, real and synthetic data present the same behaviour, with the well-known V-shaped pattern for all three jerks. For SIT observatory, in Northern America, the V-shaped is less accentuated; however, two on three expected jerks have been detected.

The results obtained for these six chosen observatories, applying different techniques (i.e. wavelet analysis or linear approximation) are summarized in Table 2. The dichotomy noted by Alexandrescu et al. (1996) in the occurrence dates of jerks, is confirmed: in the Southern hemisphere the first two jerks seem to occur clearly later than in the Northern hemisphere. For the last jerk, we are not able to conclude about a such ordering, as the available series, mainly for the Southern hemisphere, are not long enough. With the available series, we observed an intriguing spatio-temporal behaviour, with short-time details, before and after 1991.
The above examples indicate some limitations of the linear approximation method in detecting jerks, comparing with some other methods proposed by Alexandrescu et al. (1996), or Nagao et al. (2002). However, this linear approximation technique is less complicated, perfectly agrees with the common definition of jerks, and for synthetic data, as those obtained with the CM4 model, the knots of the temporal splines used in inversion scheme modeling are not detected (as in the wavelet analysis are).

\subsection{Global analysis}

The same linear approximation method is applied for a global analysis. We consider the whole Earth gridded by $1^{\circ} \times 1^{\circ}$. In each obtained location at the Earth's surface, the same approximation by straight-line segments is applied on $\dot{Y}$ component of synthetic data. Figure 5 shows a region of the Earth, situated between $60^{\circ} \mathrm{N} / 60^{\circ} \mathrm{S}$ and $80^{\circ} / 120^{\circ} \mathrm{E}$, with plots corresponding to every $20^{\circ}$ location. One of the best detection of jerks is shown in the graph corresponding to $40^{\circ} \mathrm{N} / 120^{\circ} \mathrm{E}$, which clearly indicates the three events. In some other plots only two events are clearly detected $\left(0^{\circ} / 120^{\circ} \mathrm{E}\right)$, or even only one $\left(40^{\circ} \mathrm{S} / 120^{\circ} \mathrm{E}\right)$. These examples also indicate that some events either do not appear in CM4 model, or are not clearly detected. This remark is supported by previous results when observatory data have been analyzed (see again Fig. 4). We can define some "blind" zones, where jerks are not detected. In order to delimit these areas we computed the difference $\delta=a_{2}-a_{1}$, for each $t_{0}$. The obtained range for $\delta$ is $\pm 15 \mathrm{nT} / \mathrm{yr}^{2}$, with a zero-line indicating no change in the straight-line segments tendency. We considerer that $10 \%$ of this extrema interval represents a reasonable limit in defining zones with no change or too small changes in the straight-line segments tendency. The 
$80^{\circ}$ Lon. East
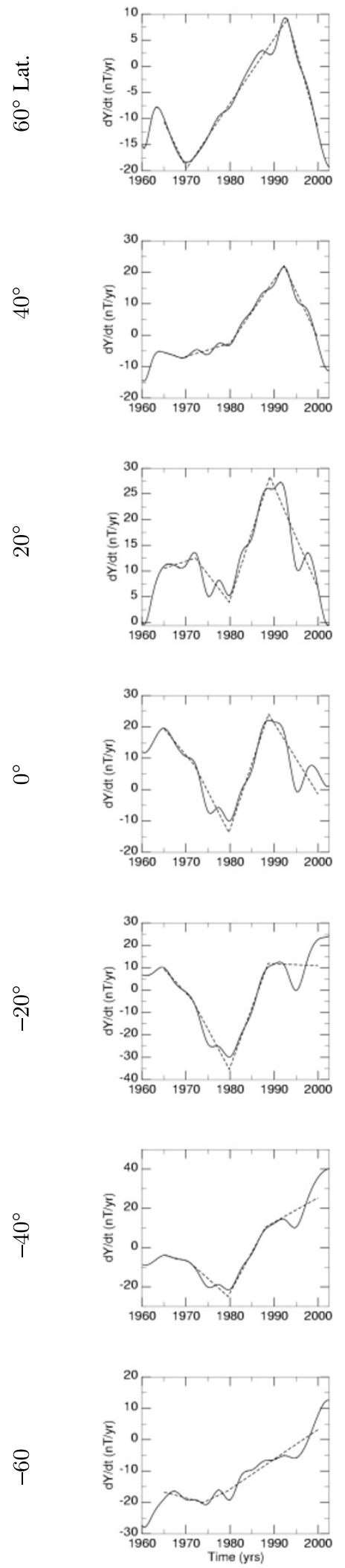

$100^{\circ}$ East

$120^{\circ}$ East
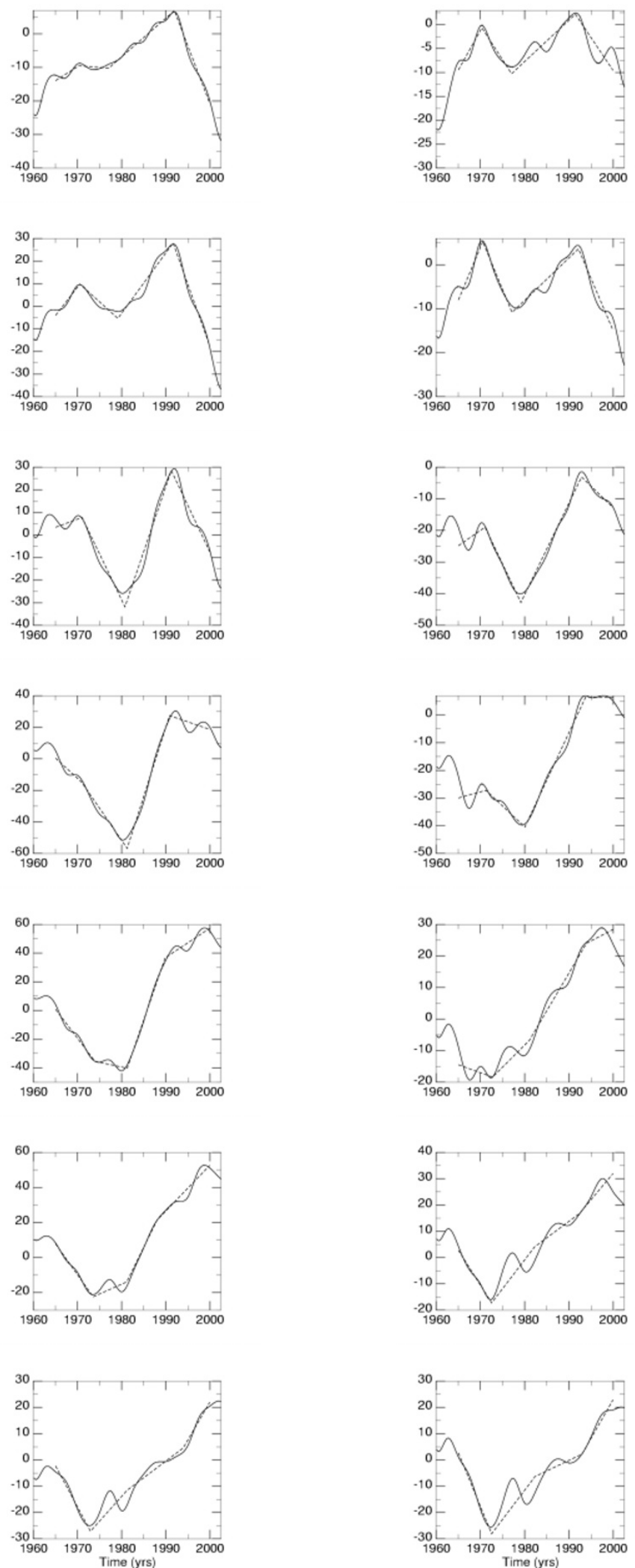

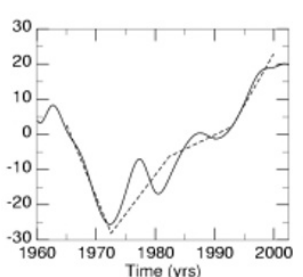

Fig. 5. Secular variation for $Y$ component at some location points: linear approximation (dashed curve) of synthetic data given by the CM4 model (solid curve). 


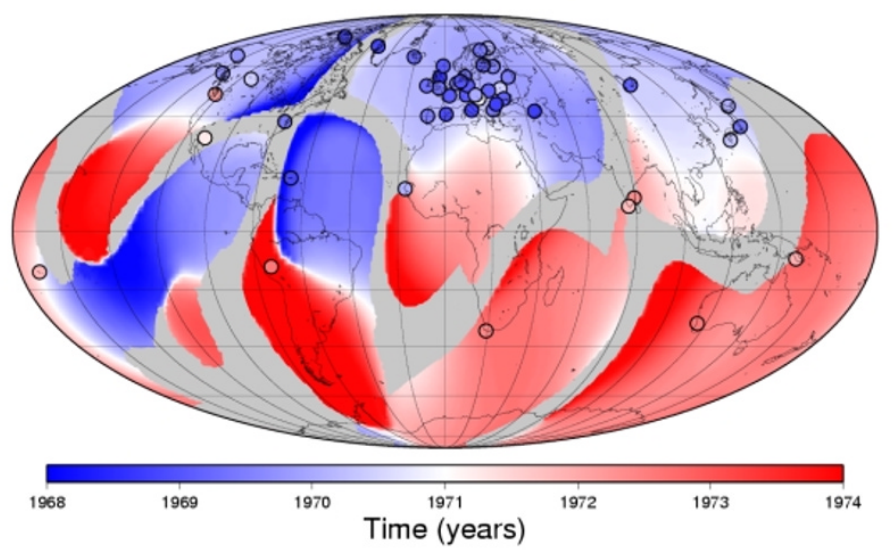

Fig. 6. Occurrence dates obtained from the CM4 synthetic data for the geomagnetic jerk centered on 1971. The same scale is used for dates given by wavelet analysis on observatory monthly mean series (full colored circle). The shadowed areas indicate the so-called "blind" zones where no jerk is detected.

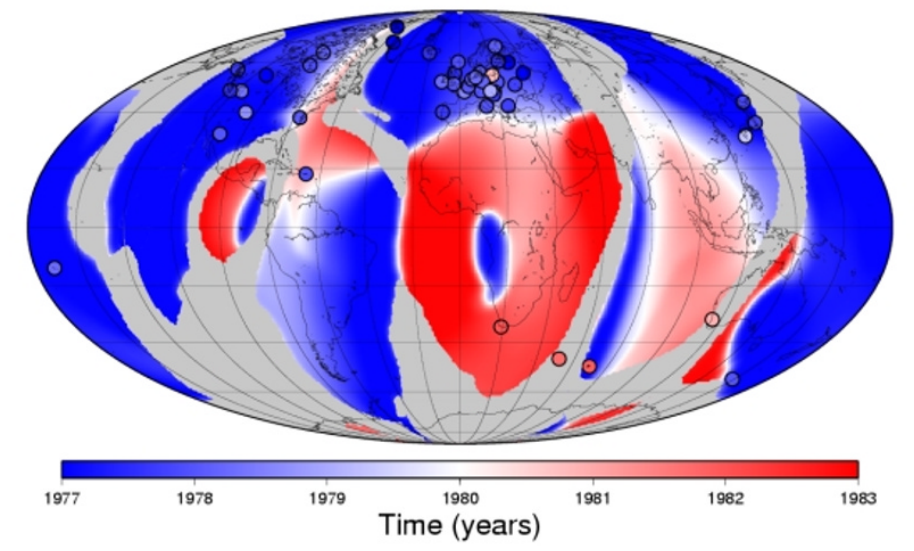

Fig. 7. Occurrence dates obtained from the CM4 synthetic data for the geomagnetic jerk centered on 1980. The same scale is used for dates given by wavelet analysis on observatory monthly mean series (full colored circle). The shadowed areas indicate the so-called "blind" zones where no jerk is detected.

areas characterised by values of $-1.5 \mathrm{nT} / \mathrm{yr}^{2}<\delta<1.5$ $\mathrm{nT} / \mathrm{yr}{ }^{2}$ are defined as "blind" zones.

The final result of the present study is presented in Figs. 6, 7 and 8 . For each interval $\left[t_{\text {begin }}, t_{\text {end }}\right]$ the dates detection has been done automatically. The three obtained maps show the occurrence dates for the events centered on 1971, 1980 and 1991. On these maps the geomagnetic jerk dates obtained from real observatory monthly means when wavelet analysed, are also plotted. The number of observatories for which the dates have been obtained is 48 for the first event, 46 for the second, and 34 for the third one. Generally speaking, for all three events, the dates obtained from observatory monthly means using wavelet analysis agree with those attributed to the respective area using the linear approximation method. Some differences of \pm 1 year ap- pear. This is not surprising as monthly means are used and two different methods applied.

The global distribution for the first geomagnetic jerk (Fig. 6) shows a clear dichotomy, separating, grossly speaking, the two hemispheres, as was previously claimed (Alexandrescu et al., 1996; De Michelis et al., 1998). The gray shadowed areas underline the missing information about occurrence dates, indeed the above defined "blind" zones. For the second event, the occurrence dates seem to be much more scattered, defining a few regions (Fig. 7). However, a general separation appears between two large areas, one being centered over the African continent. More surprising is the last map (Fig. 8), where the dates seem to indicate a change in occurrence tendency, with an early occurrence in so-called "European/African" region. How- 


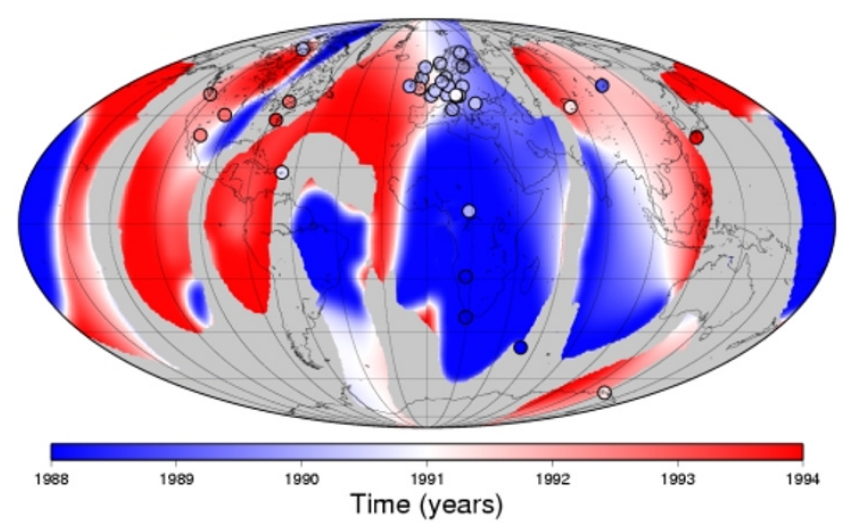

Fig. 8. Occurrence dates obtained from the CM4 synthetic data for the geomagnetic jerk centered on 1991. The same scale is used for dates given by wavelet analysis on observatory monthly mean series (full colored circle). The shadowed areas indicate the so-called "blind" zones where no jerk is detected. N.B. This jerk is more complicated in shape than the previously two.

ever, this last result must be carefully considered. The 1971 and 1980 jerks are simple in shape. The 1991 ones is more complicated in shape, with, for example, two maxima in the general secular variation trend for Hermanus observatory (one of the important one in the area). These maxima can be seen just before and after 1991. The same is observed in Fig. 5 for the synthetic curves corresponding to the Southern hemisphere.

\section{Conclusions}

The CM4 model embodies our most detailed knowledge of how the geomagnetic field has varied in both space and time during the last four decades. The information it contains has, however, not yet been fully exploited. In particular, little attention has been paid to the secular variation description associated with this model. However, the authors of CM4 model, emphasize the advantage of the strengths of their model in jerk studies (Sabaka et al., 2004). Here, we investigate the behavior of the CM4 comprehensive model since 1960 and determined changes in the secular variation trend, showing a clear evidence for geomagnetic jerks. The inclusion of Ørsted vector and scalar data, and CHAMP scalar data in the CM4 model allowed to analyse the 1991 geomagnetic jerk. In this study the geomagnetic jerks do not appear in some areas, perhaps because (1) they might not have been worldwide in occurrence or (2) the model contains limited spread data over the four decades beyond the existing observatory data. We also estimated occurrence dates for geomagnetic jerks detected in Sabaka et al. (2004) model. This result could be obtained because the CM4 model is able to separate the various field sources.

The geomagnetic jerk occurrences are highly asymmetric in their geographical distribution. This "asymmetric" behaviour, already underlined by Alexandrescu et al. (1996) and De Michelis et al. (1998) is far from simple. The obtained results clearly show that these events are not simultaneous. The occurrence dates for the 1991 jerk are-for the very dynamic region centered over African continent- difficult to be very well determined. Once more, we admit that the secular variation is much more phenomenologically rich than geomagnetic jerks.

Geomagnetic jerks, influencing the secular variation years or even decades after they have occurred, are usually quite difficult to observe. Up to now, they were detected only in observatory data. Recently, we have shown that the north magnetic pole position, which involves the field as a whole, and its velocity provides a nice indicator of jerks (Mandea and Dormy, 2003). The geomagnetic jerks themselves are an excellent tool to constrain the lower mantle conductivity (Mandea Alexandrescu et al., 1999), or to estimate its possible laterally heterogeneous conductivity (Nagao et al., 2003). Moreover, the observed patterns, with zones characterized by different occurrence epochs or zones where no jerks have been detected in the CM4 model, may stimulate the discussion of the role of torsional oscillations (Bloxham et al., 2002).

To investigate the global or local character of geomagnetic jerks, and to better understand each of those phenomena and how they relate to one another, an improvement in measurement accuracy and resolution, in space and time, is needed. Presently, the geomagnetic field is monitored from space by a fleet of dedicated satellites, Ørsted, CHAMP and SAC-C. The secular variation at decadal scale or longer, however, cannot be well determined due to the fairly short life-time of these missions. An important question, therefore, is how well do secular changes derived from satellite data fit the ground-based measurements, and how geomagnetic jerks can be detected in satellite data. By ensuring long-term space observations with a better spatial resolution the multi-satellite Swarm mission ${ }^{2}$ (Friis-Christensen et al., 2004), scheduled for launch in 2009, will improve secular variation models all over the Globe. And, if a geomagnetic jerk occurs during the course of satellite missions

${ }^{2}$ http://www.esa.int/esaLP/swarm.html 
its characteristics could be investigated in much more detail than was previously possible with just ground-based data or global models.

Acknowledgments. All maps were plotted using the GMT software (Wessel and Smith, 1991). We would like to thank Hiromichi Nagao and Terence Sabaka for useful suggestions that helped in improving the paper. We would also like to thank Satoshi Kanai for his editorial work. This is IPGP contribution 2024.

\section{References}

Alexandrescu, M., D. Gibert, G. Hulot, J.-L. Le Mouël, and G. Saracco, Detection of geomagnetic jerks using wavelet analysis, J. Geophys. Res., 100, 12557-12572, 1995.

Alexandrescu, M., D. Gibert, G. Hulot, J.-L. Le Mouël, and G. Saracco, Worldwide analysis of geomagnetic jerks, J. Geophys. Res., 101, 21975-21994, 1996.

Bloxham, J., S. Zatman, and M. Dumberry, The origin of geomagnetic jerks, Nature, 420, 65-68, 2002.

Chau, H. D., J. Ducruix, and J.-L. Le Mouël, Sur le caractère planétaire du saut de variation séculaire de 1969-1970, C. R. Acad. Sci. B, 298, 157-160, 1981.

Courtillot, V., J. Ducruix, and J.-L. Le Mouël, Sur une accélération récente de la variation séculaire du champ magnétique terrestre, C.R. Acad. Sci. D, 287, 1095-1098, 1978.

De Michelis, P., L. Cafarella, and A. Meloni, Worldwide character of the 1991 jerk, Geophys. Res. Lett., 25, 377-380, 1998.

Friis-Christensen, E., A. De Santis, A. Jackson, G. Hulot, A. Kuvshinov, H. Lühr, M. Mandea, S. Maus, N. Olsen, M. Purucker, M. Rothacher, T. Sabaka, A. Thomson, S. Vennerstrom, and P. Visser, Swarm. The Earth's Magnetic Field and Environment Explorers, ESA SP-1279(6), 2004.

Jackson, A., A. Jonkers, and M. Walker, Four centuries of geomagnetic secular variation from historical records, Phil. Trans. R. Soc. Lond., 358, 957-990, 2000.

Langel, R. A., T. J. Sabaka, R. T. Baldwin, and J. A. Conrad, The nearEarth magnetic field from magnetospheric and quiet-day ionospheric sources and how it is modeled, Phys. Earth Planet Inter., 98, 235-267, 1996.

Le Huy, M., M. Alexandrescu, G. Hulot, and J.-L. Le Mouël, On the characteristics of successive geomagnetic jerks, Earth Planets Space, 50, 723-732, 1998.

Mandea, M. and E. Dormy, Asymmetric behavior of magnetic dip poles, Earth Planets Space, 55, 153-157, 2003.

Mandea, M. and B. Langlais, Observatory crustal magnetic biases during MAGSAT and Ørsted satellite missions, Geophys. Res. Lett., 29, 10.1029/2001GL013693, 2002.

Mandea Alexandrescu, M., D. Gibert, J.-L. Le Mouël, G. Hulot, and G. Saracco, An estimate of average lower mantle conductivity by wavelet analysis of geomagnetic jerks, J. Geophys. Res., 104, 17735-17745, 1999.

Mandea, M., E. Bellanger, and J.-L. Le Mouël, A geomagnetic jerk for the end of 20th century?, Earth Planet. Sci. Lett., 183, 369-373, 2000.

Nagao, H., T. Iyemori, T. Higuchi, S. Nakano, and T. Araki, Local time features of geomagnetic jerks, Earth Planets Space, 54, 119-131, 2002.

Nagao, H., T. Iyemori, T. Higuchi, and T. Araki, Lower mantle conductivity anomalies estimated from geomagnetic jerks, J. Geophys. Res., 108(B5), 2254, doi:10.1029/2002JB001786, 2003.

Sabaka, T. J. and R. T. Baldwin, Modeling the Sq magnetic field from POGO and MAGSAT satellite and contemporaneous hourly observatory data, HSTX/G\&G-9302, Hughes STX Corp., 7701 Greenbelt Road, Greenbelt, $M D, 1993$.

Sabaka, T., N. Olsen, and R. A. Langel, A comprehensive model of the quiet-time, near-Earth magnetic field: phase 3, Geophys. J. Int., 151, 32-68, 2002.

Sabaka, T., N. Olsen, and M. E. Purucker, Extending comprehensive models of the Earth's magnetic field with Ørsted and CHAMP data, Geophys. J. Int., 159, 521-547, 2004.

Stewart, D. N. and K. Whaler, Geomagnetic disturbance fields: An analysis of observatory monthly means, Geophys. J. Int., 108, 215-223, 1992.

Wessel, P. and W. H. F., Smith, Free software helps map and display data. EOS Trans. AGU, 72, 445-446, 1991.

A. Chambodut and M. Mandea (e-mail: mioara@gfz-potsdam.de) 